\title{
SISTEMÁTICA PARA O GERENCIAMENTO DE RISCOS EM \\ LABORATÓRIOS ABERTOS NA FASE DE OPERAÇÃO
}

Rayane Silva (rayanebatista.ep@gmail.com) - Faculdade de Tecnologia/Universidade de Brasília

Viviane Vasconcellos Ferreira Grubisic (vivianegrubisic@unb.br) - Faculdade de Tecnologia / Universidade de Brasília

Andrea Cristina dos Santos (andreakieck@gmail.com) - Faculdade de

Tecnologia/Universidade de Brasília

\section{RESUMO}

O presente artigo apresenta uma sistemática para o gerenciamento de riscos voltada para laboratórios abertos na fase de operação. O objetivo da sistemática é organizar em uma sequência lógica e estruturada em fases, atividades e tarefas o conhecimento necessário para identificar, analisar e tratar os riscos que possam surgir ao longo da fase de operação de um laboratório aberto (LA). Também, para cada processo foram propostas ferramentas para auxiliar na execução da sistemática. Primeiramente, foi feita uma revisão da literatura sobre LA e, principalmente sobre gerenciamento de riscos. Após o desenvolvimento da sistemática, a mesma foi submetida para à avaliação por parte de colaboradores do Laboratório Aberto da Faculdade de Tecnologia, da Universidade de Brasília (UnB). A sistemática foi aplicada e avaliada por eles e a partir desses resultados, pode-se inferir que a implementação da sistemática foi bem-sucedida, se provando uma boa alternativa para auxiliar no gerenciamento de riscos em laboratórios abertos.

Palavras chave: gerenciamento de riscos, laboratório aberto, operação. 


\section{INTRODUÇÃ̃}

No contexto da Universidade existem diversos tipos de laboratórios que apoiam as atividades de ensino, pesquisa e extensão. Uma das modalidades é chamada de Laboratório Aberto (LA), uma categoria relativamente nova nas universidades que tem uma proposta diferenciada de abrir as portas para a sociedade. $\mathrm{O}$ ambiente de um LA proporciona uma aprendizagem de forma colaborativa no desenvolvimento de novos produtos, processos e negócios com foco principal na economia criativa e inovação (SANTOS, et al., 2017).

O termo LA surgiu por meio de uma adaptação do conceito dos laboratórios de fabricação digital - FabLab que podem ter uma vertente acadêmica, profissional ou pública, criado pelo Massachusetts Institute of Technology (MIT) (MIKHAK, et al., 2002). No Brasil, através do Projeto SIBRATECSHOP de 2015, a parceria entre o MCTI (Ministério da Ciência, Tecnologia, Inovações), SENAI (Serviço Nacional de Aprendizagem Industrial) e SEBRAE (Serviço Brasileiro de Apoio às Micro e Pequenas Empresas) objetivou apoiar novas empresas e fomentar o empreendedorismo e a inovação. "Foram inicialmente implementados 13 LAs, sendo somente dois em Universidades Federais (UFRGS e ITA), e o restante nas unidades do SENAI distribuídas no país" (SANTOS, et al., 2017). Na Faculdade de Tecnologia (FT) da Universidade de Brasília (UnB) existe o Laboratório Aberto de Brasília, cuja implantação foi promovida por meio da parceria entre a FINATEC (Fundação de Empreendimentos Científicos e Tecnológicos), SENAI - Direção Nacional, MCTI e a própria UnB.

O objetivo principal do LAB da FT é de contribuir com a formação de um engenheiro empreendedor, por meio de um espaço físico compartilhado que contenha equipamentos e facilidades para o desenvolvimento de soluções tecnológicas e novos negócios (SANTOS, et al., 2017). A criação de um laboratório aberto passa por uma fase de planejamento na qual estudos como de viabilidade técnica, econômico-financeira, ambiental e de desenvolvimento de serviços, são elaborados e avaliados. Após a finalização destes estudos, tem-se a fase de implementação na qual o planejamento, sistematização e trabalho em equipe envolvendo todas as partes interessadas (discentes, docentes e comunidade) são colocados em prática (SANTOS, et al., 2017).

Por fim, inicia-se a fase de operação de um LA que consiste na realização das atividades diárias, ou seja, reuniões de desenvolvimento e prototipagem de projetos de engenharia. Porém, 
essa fase pode estar sujeita a riscos, que precisam ser gerenciados de forma estruturada a fim de garantir a existência e o alcance dos objetivos de um Laboratório Aberto.

No Brasil, a cultura de gerenciar riscos e oportunidades não tem sido internalizada pelas empresas, está espalhada em conceitos desatualizados, e quando utilizada, é normalmente através da contratação de serviços especializados externos (BARALDI, 2018).

Dentro deste contexto, uma sistemática de gerenciamento de riscos (GR) voltado para a fase de operação de um LA tende a contribuir para a identificação, análise e tratamento dos riscos.

Como principais benefícios de um modelo de GR podem-se citar: a melhora da identificação de oportunidades e ameaças, prevenção de perdas, gestão de incidentes, melhora da eficácia e eficiência operacional, aumento da resiliência da organização (ABNT, 2009). Assim, o presente trabalho tem como objetivo propor uma sistemática para o GR voltado para fase de operação de LA. 


\section{REVISÃO BIBLIOGRÁFICA}

\subsection{Laboratório Aberto (LA)}

Wilczynski et al. (2016), conforme citado por Zimmermann (2018), descreve os makerspaces (também chamados de FabLabs, laboratórios abertos entre outros nomes) como espaços físicos onde seus usuários podem utilizar ferramentas de fabricação digital e equipamentos eletrônicos para criar, projetar e produzir novos produtos. Segundo Zimmermann (2018), durante a execução da etapa de geração e seleção de ideias para definição do pacote de serviços do LAB, padrões de serviços foram identificados dentre os diversos LAs pesquisados. Estas atividades são geralmente organizadas da seguinte forma:

- Célula de marcenaria;

- Célula de eletrônica;

- Célula de manufatura aditiva;

- Coworking.

Atrelado à organização de um LA, tem-se os serviços prestados por ele. Segundo Zimmermann (2018), os serviços prestados pelo LAB são:

- Desenvolvimento de protótipos - consiste em projetar, modelar, fabricar e realizar o acabamento de um protótipo.

- Acesso à infraestrutura laboratorial - disponibilizar equipamentos e ferramentas para usufruto do usuário (desde que comprovada a competência necessária)

- Capacitação - eventos, cursos, oficinas e workshops oferecidos pelo LAB.

Com relação aos stakeholders de um LA, Zimmermann (2018) propõe um mapa de stakeholders (Figura 1) onde eles são classificados em 5 grupos: (i) provedores de serviço, (ii) clientes, (iii) parceiro, (iv) órgãos reguladores e (v) fornecedores. 


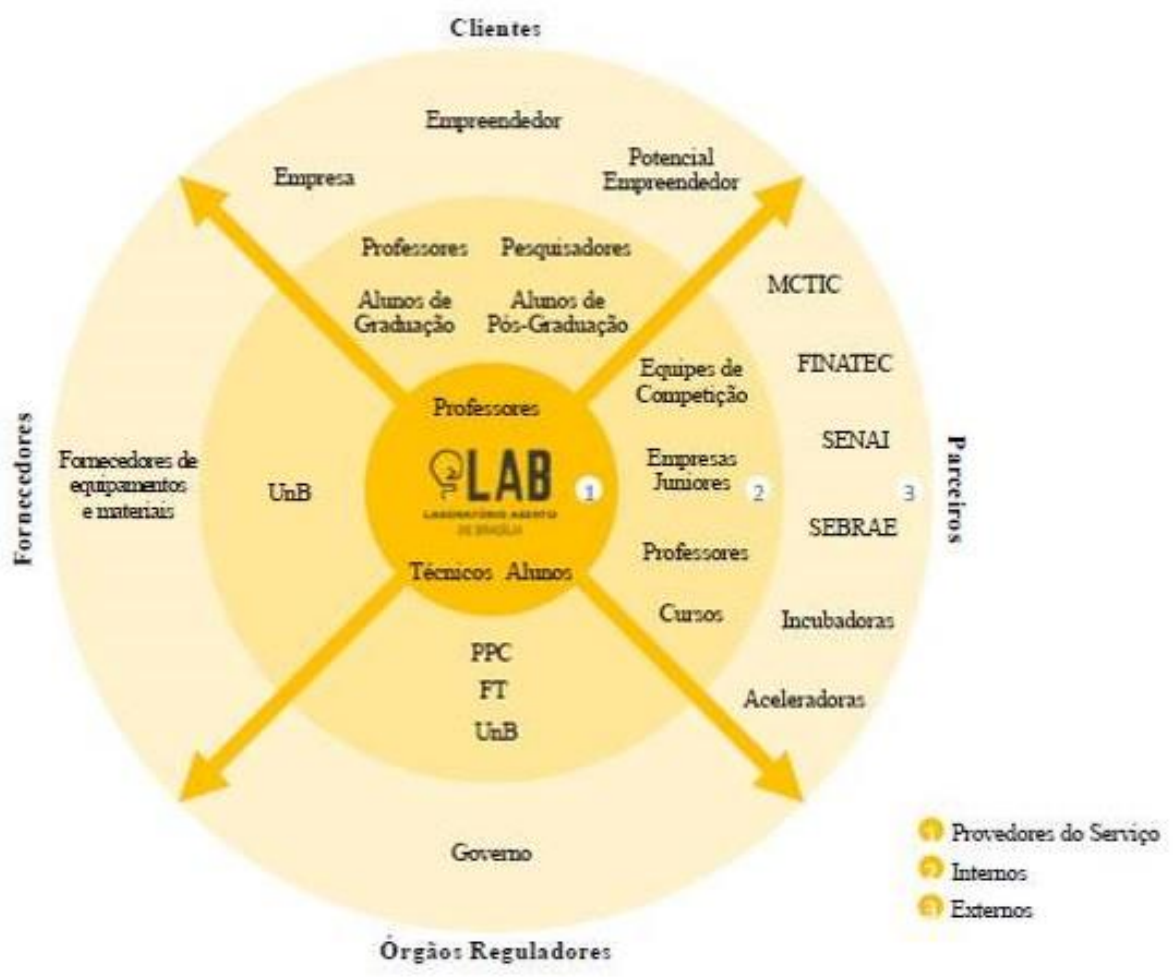

Figura 1 - Mapa de Stakeholders Fonte: (ZIMMERMANN, 2018)

- Provedores de serviço - pessoas que entregam a proposta de valor do LAB;

- Clientes - usuários do serviço do LAB;

- Parceiros - parceiros internos (suporte para a captação e execução de projetos) e externos (fornecem recursos para implementação e operação) do LAB;

- Órgãos reguladores - normas e legislação que regulamentam o LAB;

- Fornecedores - fornecedores internos e externos do LAB.

\subsection{Riscos}

Segundo a ISO 31000 (2009), risco é definido como o "efeito da incerteza nos objetivos", ou seja, é uma associação das consequências de um evento com suas respectivas probabilidades de ocorrência. Sob uma perspectiva semelhante, Prince 2 (2002) e PMI (2017) trazem definições análogas mais detalhada, onde risco é um conjunto de eventos ou uma condição incerta que, caso ocorram, terão efeitos sobre os objetivos do projeto. Este efeito pode ser positivo, definido como oportunidade, ou negativo, definido como ameaça. 
De maneira diferenciada, COSO (2017) trata do risco como a possibilidade em que um evento aconteça, afetando de forma negativa a realização dos objetivos da organização. Ou seja, COSO (2017) adota uma perspectiva somente negativa quando se trata do termo risco.

\subsection{Gerenciamento de riscos}

Diversos autores apresentam modelos para o gerenciamento de riscos, mas, basicamente, todas as definições convergem para o mesmo ponto: conhecer, avaliar e tratar os eventos incertos que cercam os objetivos de uma organização. Neste trabalho, quatro modelos de gerenciamento de riscos serão destacados: a norma NBR ISO 31000 (2009), PRINCE 2 (2002), PMI (2017) e COSO (2017).

Dentre eles, tem-se respectivamente, uma norma técnica nacional com uma abordagem mais genérica sobre o GR, dois modelos voltados para o GR em projetos, focadas em boas práticas área e por fim, tem-se um modelo com enfoque no mundo corporativo, mostrando as formas mais adequadas de se abordar riscos dentro das organizações. A Figura 2 apresenta um resumo desses modelos com seus processos constituintes. 
PRINCE II (2002)

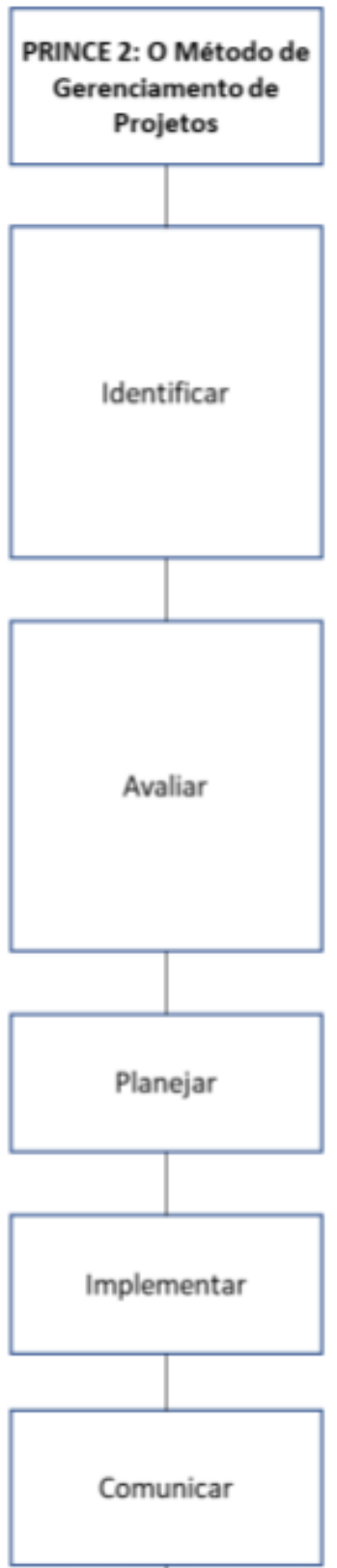

ABNT (2009)
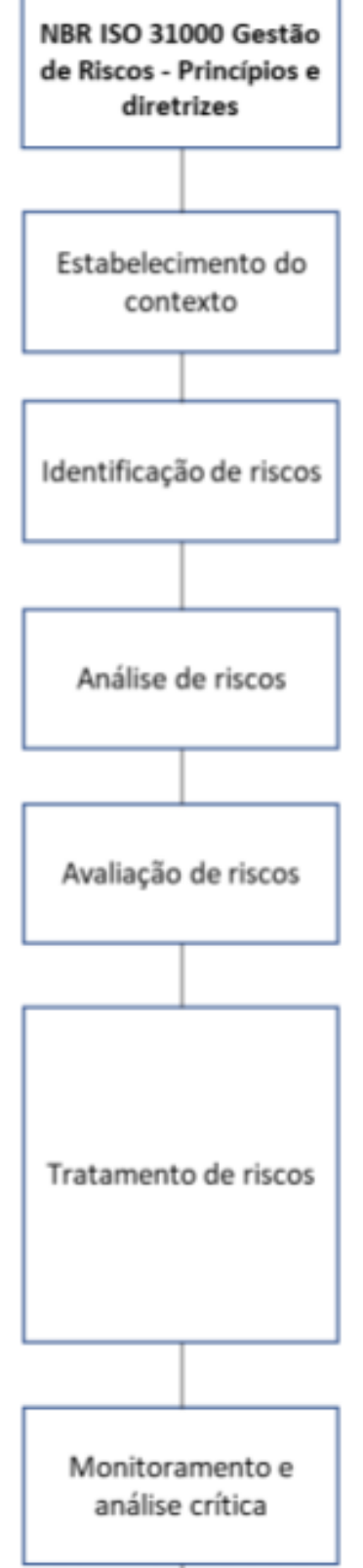

PMI (2017)

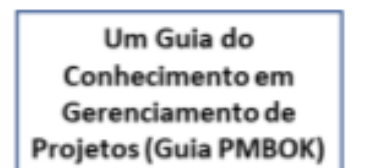

Projetos (Guia PMBOK)

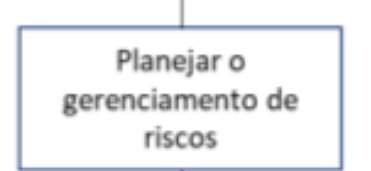

Realizar a análise qualitativa dos riscos

Realizar a análise quantitativa dos riscos

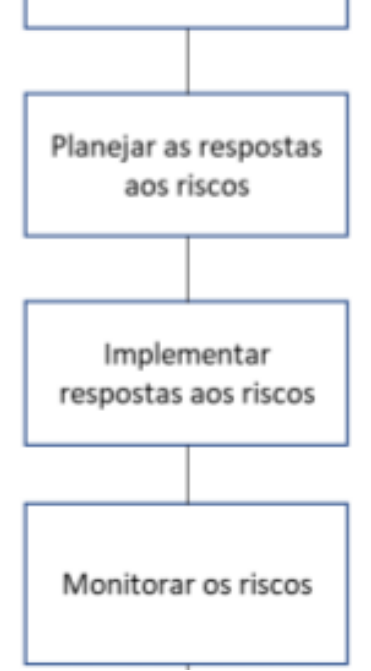

$\cos 0(2017)$

coso: Gerenciamento de Riscos Corporativos - Estrutura Integrada
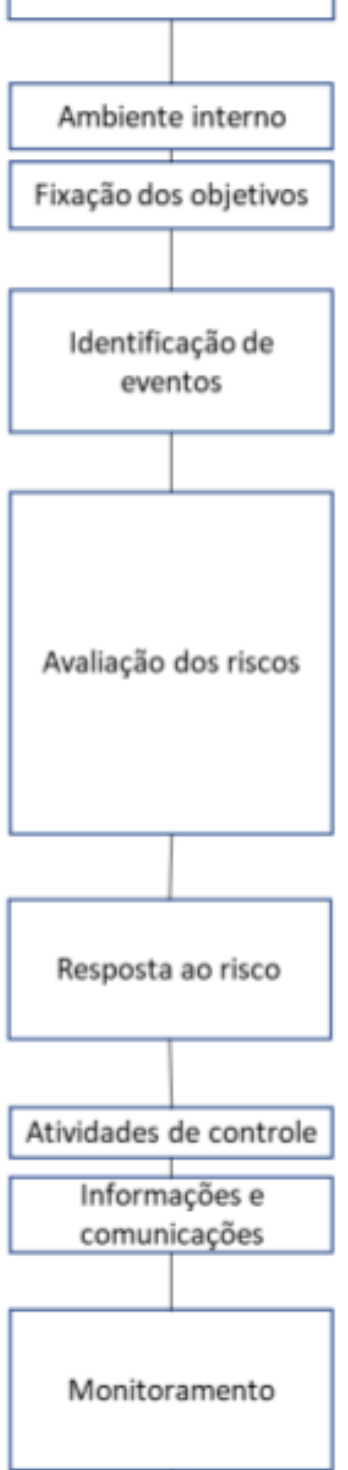

Figura 2- Modelos gerais de gerenciamento de riscos. Fonte: Autores.

Comparativamente, os processos são similares e indicam caminhos essenciais para um bom plano: a identificação dos riscos, seguida da análise, o planejamento das respostas e por fim, o controle e monitoramento. Adotando esses processos, os riscos podem ser identificados, analisados, tratados, monitorados e controlados. 
A identificação de riscos consiste no processo de levantamento dos riscos e descrição das suas causas e consequências (ameaças e oportunidades) que possam afetar qualquer objetivo de uma organização (PMI, 2017).

Por sua vez, a análise dos riscos busca compreender a natureza dos riscos em termos de sua probabilidade de ocorrência e do impacto que terá sobre os objetivos de uma organização e determinar o nível de cada risco (ABNT, 2009).

O tratamento dos riscos é o processo de modificação dos riscos avaliados, onde ações são desenvolvidas para que ocorra uma mudança efetiva na probabilidade de ocorrência dos riscos e/ou nos seus impactos (ABNT, 2009).

Por fim, o monitoramento e controle dos riscos é a etapa responsável pela verificação, supervisão e dimensionamento de tudo que foi e está sendo realizado no âmbito da gestão de riscos (ABNT, 2009).

\subsection{Sistemática de GR}

A sistemática proposta constitui-se de fases, atividades, tarefas e ferramentas para o GR em LA, tendo como base o planejamento, identificação, análise e tratamento dos riscos. Primeiramente, foi desenvolvida uma representação gráfica da sistemática conforme apresentada na Figura 3.

A fase a ser desenvolvida é representada por um único pentágono no qual aparece o nome da fase, sendo esse desdobrado em atividades representadas por quadrados. Por fim, temse a representação da saída da fase, contendo o principal resultado gerado pelas atividades. 
NOME DA FASE

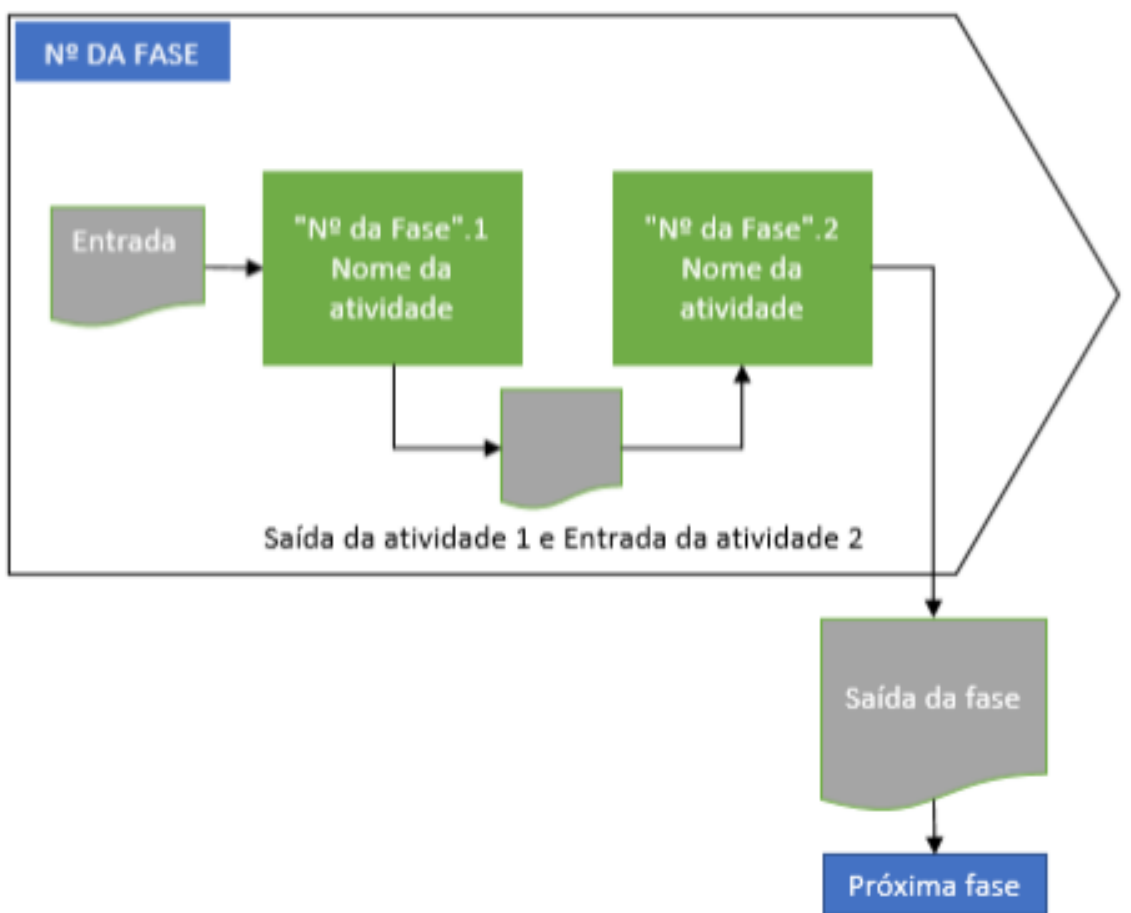

Figura 3 - Representação genérica das fases e atividades da sistemática. Fonte: SILVA, 2019.

Com base na Figura 3, foi proposta uma representação contendo as quatro fases da sistemática para o Gerenciamento de Riscos em LAs com suas respectivas atividades, conforme ilustra a Figura 4. 


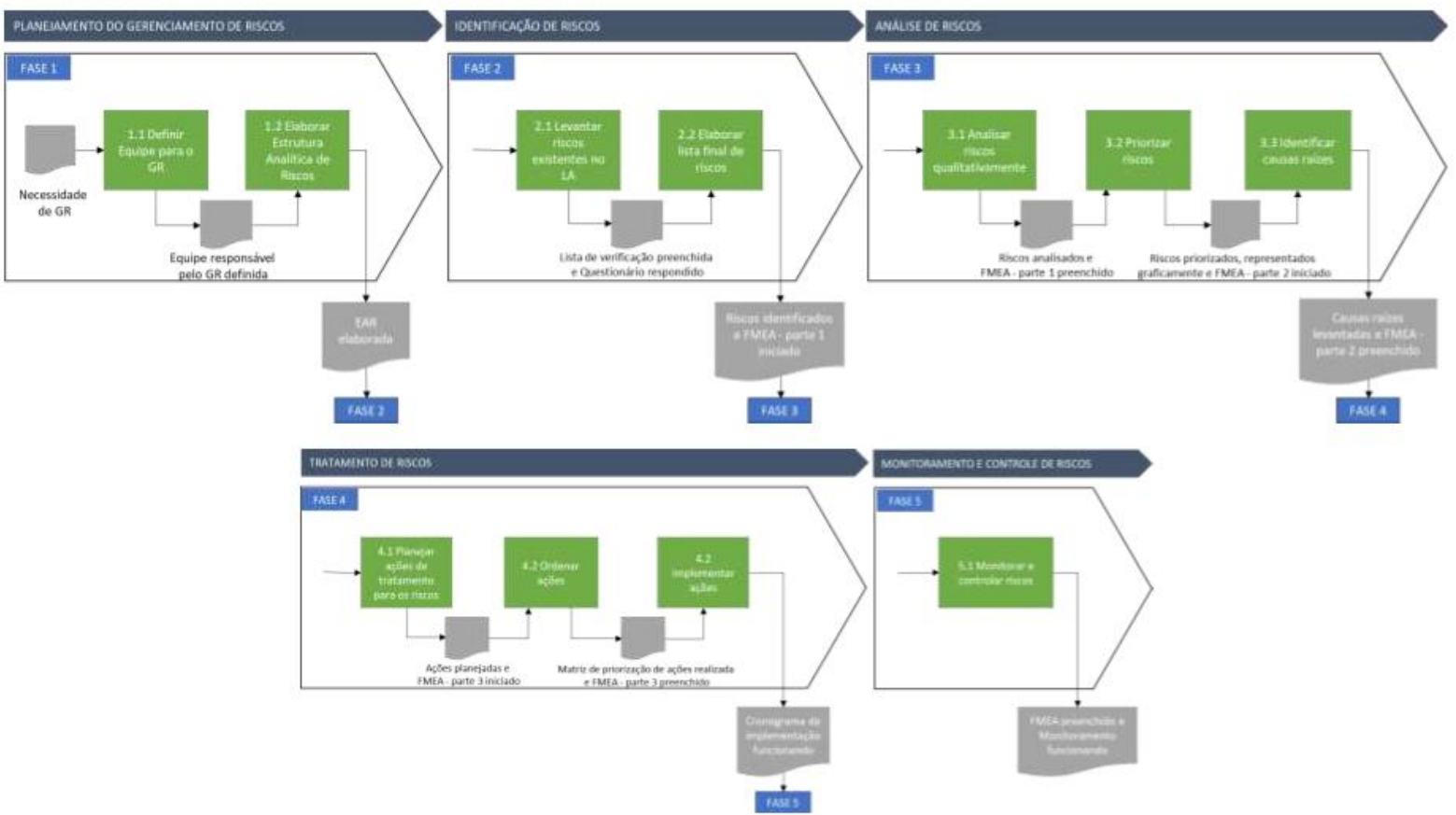

Figura 4 - Representação das fases e atividades da sistemática. Fonte: SILVA, 2019.

A seguir o detalhamento da metodologia proposta.

\subsection{Fase1 - Planejamento do Gerenciamento de Riscos}

O planejamento do GR é a fase que inicia a sistemática, ou seja, quando se tem uma "Necessidade de um Gerenciamento de Riscos".

Para isso, são propostas duas atividades:

1.1 Definir equipe para o GR e

1.2. Elaborar Estrutura Analítica de Riscos, com suas respectivas entradas e saídas, conforme apresentado na Figura 5. 


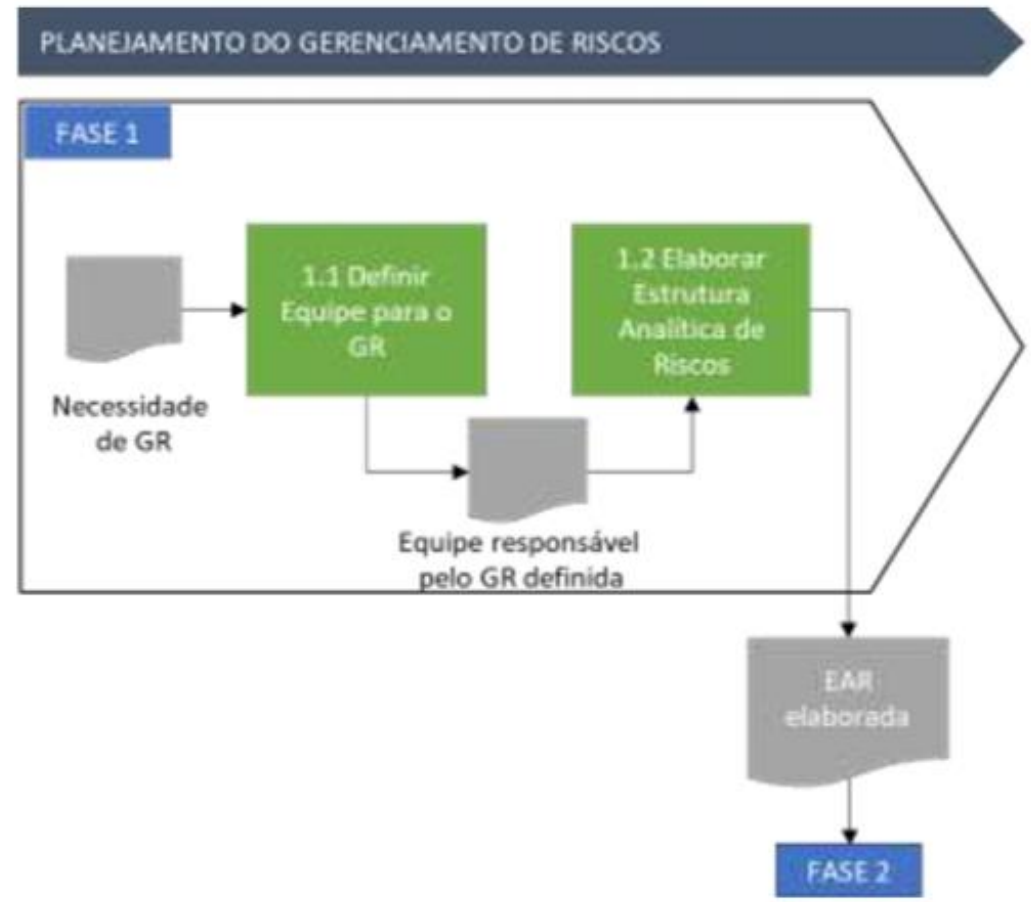

Figura 5 - Fase 1: Planejamento do Gerenciamento de Riscos. Fonte: SILVA, 2019.

Primeiramente, tem-se a atividade "Definir equipe para o GR", cuja finalidade é a seleção da equipe que será responsável pelo gerenciamento de riscos no laboratório. Para auxiliar esta seleção propõem-se um formulário de lista de colaboradores do LA e orientações fornecidas no Quadro 1.

Quadro 1 - Orientações para seleção de equipe.

\begin{tabular}{|c|l|}
\hline \multicolumn{2}{|c|}{ Orientações para seleção de equipe } \\
\hline 1 & Colaborador com experiência superior à no mínimo 1 ano no LA \\
\hline 2 & Professor responsável pelo LA \\
\hline 3 & Colaborador que exerça cargo de gerência \\
\hline 4 & Responsável pela célula de eletrônica \\
\hline 5 & Responsável pela célula de manufatura aditiva \\
\hline 6 & Responsável pela célula de marcenaria \\
\hline 7 & Responsável pelo espaço de coworking \\
\hline 8 & Representante de stakeholder externo \\
\hline
\end{tabular}

Fonte: SILVA, 2019. 
Em seguinte, tem-se a atividade 1.2 "Elaborar a Estrutura Analítica de Riscos" (EAR) que funciona como auxílio para o agrupamento e estruturação das categorias de risco que serão utilizadas no projeto. Esta atividade é desmembrada em duas tarefas:

1.2.1 - Definir o primeiro nível da EAR (divisão base de todas as fontes de risco do projeto) e

1.2.2 - Definir os demais níveis (subdivisões de cada categoria base). Uma EAR aplicada a um LA e suas subcategorias, é mostrada na Figura 6.

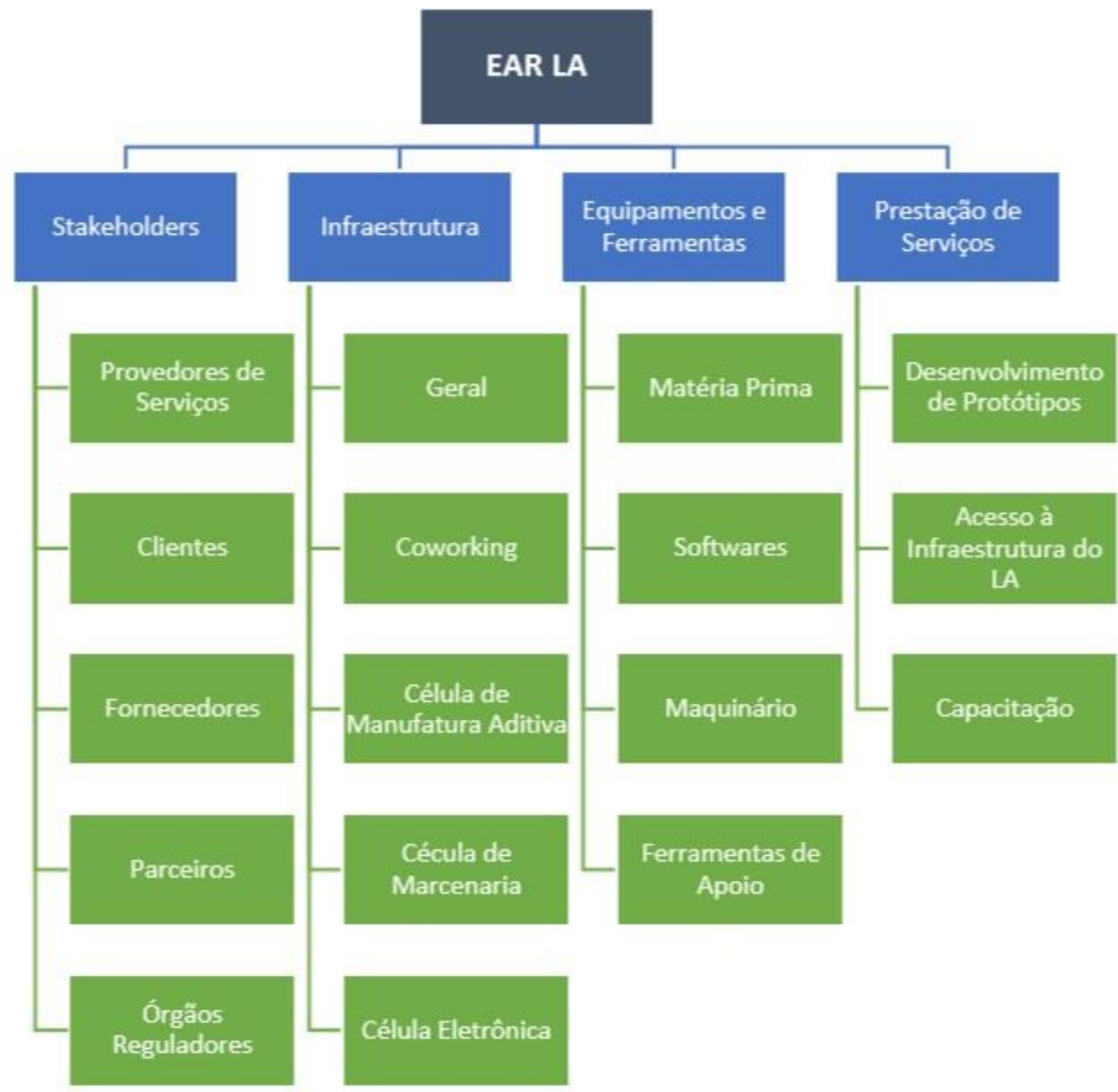

Figura 6 - EAR genérica para Laboratórios Abertos. Fonte: SILVA, 2019.

A EAR contendo as categorias e subcategorias de riscos irão formar a principal saída da Fase 1 - Planejamento do Gerenciamento de Riscos e servirão de entrada para a fase seguinte. 


\subsection{Fase 2 - Identificação de riscos}

A Fase 2 consiste na identificação de riscos existentes na operação de um LA com base nas categorias e subcategorias da EAR definida na fase anterior. Para a identificação de riscos de um LA, propõe-se duas atividades, conforme mostra a Figura 7.

\section{IDENTIFICACĀO DE RISCOS}

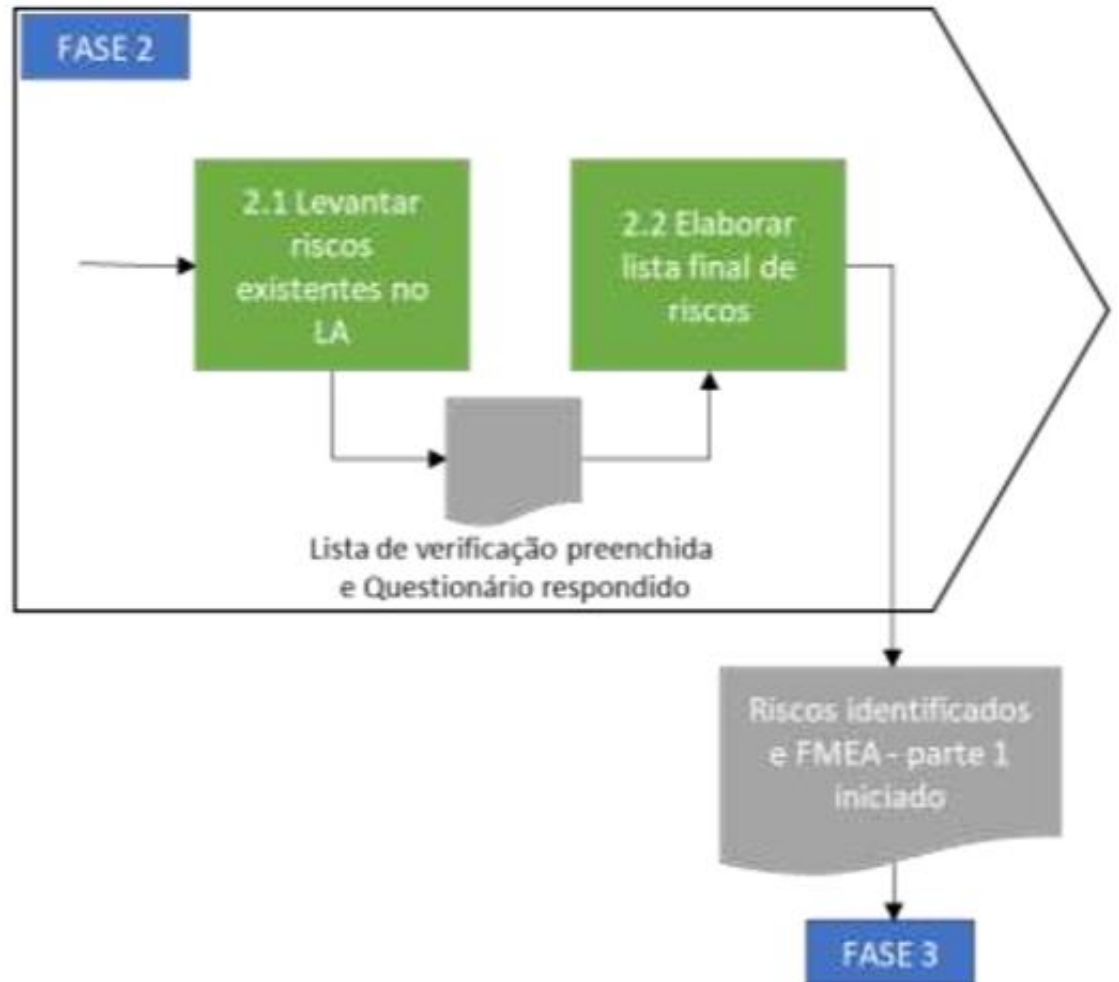

Figura 7 - Fase 2: Identificação de riscos. Fonte: SILVA, 2019.

Para a atividade 2.1 - Levantar riscos existentes no LAB foram definidas cinco tarefas:

- Tarefa 2.1.1 - Elaborar lista de verificação: esta tarefa serve para analisar se os riscos que foram elencados através de informações históricas e conhecimento acumulado dos integrantes do LA se aplicam ou não àquela realidade em questão.

- Tarefa 2.1.2 - Elaborar questionário de levantamento de riscos: essa tarefa consiste em elaborar um roteiro de entrevista que tem como objetivo o levantamento de novos riscos que a fase de operação de LA pode estar sujeita. 
- Tarefa 2.1.3 - Realizar entrevistas com os colaboradores do laboratório: esta tarefa se refere a realização da entrevista com a equipe de GR do laboratório. Sugere-se primeiramente a coleta de respostas do questionário e depois o preenchimento da lista de verificação.

Terminada a atividade de levantamento de riscos é importante que se faça uma revisão dos riscos obtidos. As seguintes tarefas são propostas para a obtenção da lista final de riscos.

- Tarefa 2.2.1 - Analisar material produzido na atividade 2.1: esta tarefa consiste na avaliação de todo o material gerado na atividade anterior (EAR, lista de verificação e respostas obtidas nas entrevistas realizadas), com o intuito de se ter conhecimento sobre todas as informações coletadas.

- Tarefa 2.2.2 - Filtrar riscos conforme a EAR: revisar e categorizar os riscos levantados de acordo com a EAR proposta.

- Tarefa 2.2.3 - Montar lista final de risco: montar a lista de riscos propriamente dita, adicionando no FMEA.

De posse do FMEA preenchido com os riscos, a fase seguinte consiste na análise dos riscos identificados.

\subsection{Fase 3 - Análise de riscos}

A presente fase consiste em examinar os riscos identificados segundo parâmetros definidos pelo método a ser utilizado FMEA (Failure Mode and Effects Analysis ou Análise dos Modos e Efeitos de Falha), a fim de ponderá-los e com isso priorizá-los de acordo com seu NPR (Número de Prioridade de Risco). Para a execução dessa fase são propostas três atividades:

(i) analisar riscos qualitativamente,

(ii) (ii) priorizar riscos e

(iii) (iii) identificar causas raízes.

Estas atividades servirão como apoio para a proposição de ações para o tratamento dos riscos. A Figura 8, retrata as entradas e saídas de cada uma dessas atividades citadas acima. 


\section{ANAULSE DE RISCOS}

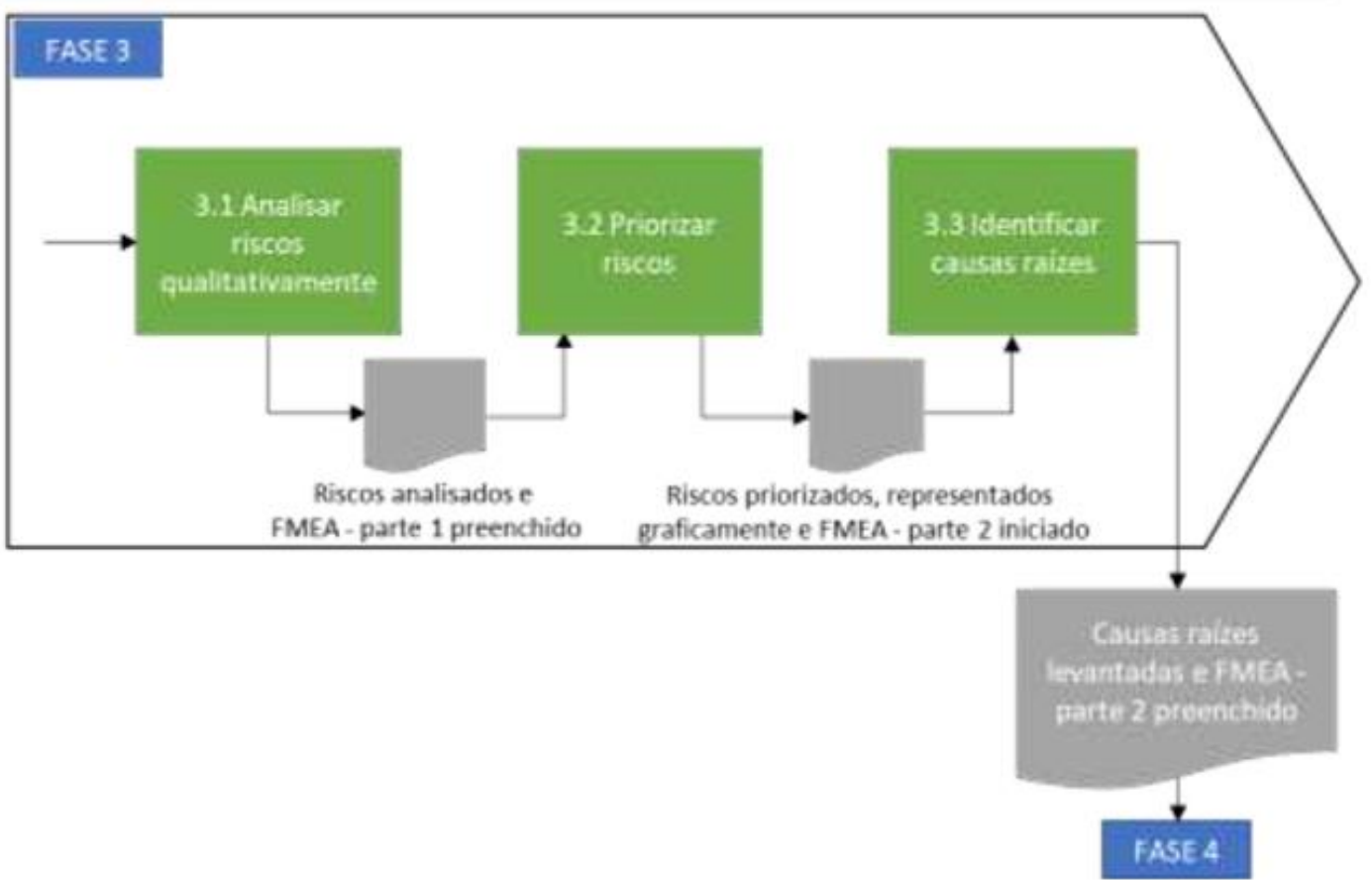

Figura 8 - Fase 3: Análise de riscos. Fonte: SILVA, 2019.

A atividade 3.1 - Analisar riscos qualitativamente é baseada na aplicação da FMEA. A FMEA tem como objetivo analisar todas as maneiras em que os riscos podem ocorrer (modos de falha), efeitos, severidade do efeito, causas dos riscos, probabilidade de ocorrência da causa, controles de prevenção e detecção, o nível de prioridade do risco, ações recomendadas, responsáveis e data para execução da ação.

Neste trabalho, os modos de falha equivalem aos riscos já identificados na Fase 2 Identificação dos riscos. Por isso, na atividade 3.1 tem-se, primeiramente, a definição dos efeitos de cada risco que compõe a lista final de riscos identificados.

Após essa consideração, propõe-se a definição dos elementos do método FMEA ao longo das três tarefas desta atividade.

- Tarefa 3.1.1 - Identificar os efeitos de cada risco e analisar suas severidades: nesta tarefa, são identificados os efeitos que cada risco pode proporcionar caso ele ocorra, como também ponderá-los segundo seu grau de severidade (S). O critério de avaliação a ser utilizado é apresentado no Quadro 2. Para os riscos que tiverem mais de um efeito, deve-se calcular a severidade média. 
Quadro 2 - Escala de severidade (S).

\begin{tabular}{|c|l|c|}
\hline \multicolumn{2}{|c}{ Efeito } & Escala \\
\hline Muito alta & Quando compromete a segurança da operação & 5 \\
\hline Alta & Quando provoca alta insatisfação do cliente & 4 \\
\hline Moderada & Quando provoca alguma insatisfação & 3 \\
\hline Baixa & Quando provoca uma leve insatisfação & 2 \\
\hline Mínima & Risco que afeta minimamente o desempenho do sistema & 1 \\
\hline
\end{tabular}

Fonte: Adaptado (FOGLIATTO, et al., 2009).

- Tarefa 3.1.2 - Identificar as causas de cada risco e analisar suas ocorrências (O):as causas que podem originar riscos são identificadas e mensuradas de acordo com sua probabilidade de ocorrência. Sugere-se o uso do critério de avaliação exposto no Quadro 3.

Quadro 3 - Escala de ocorrência (0).

\begin{tabular}{|c|l|c|}
\hline \multicolumn{2}{|l}{ Ocorrência de riscos (O) } & Escala \\
\hline Muito alta & Causas quase inevitáveis & 5 \\
\hline Alta & Causas ocorrem com frequência & 4 \\
\hline Moderada & Causas ocasionais & 3 \\
\hline Baixa & Causas raramente ocorrem & 2 \\
\hline Mínima & Causas muito improváveis & 1 \\
\hline
\end{tabular}

Fonte: Adaptado (FOGLIATTO, et al., 2009).

- Tarefa 3.1.3 - Identificar os métodos de detecção (D) dos riscos e analisá-los: consiste na identificação dos métodos de detecção (D) já adotados pelo o laboratório para evitar as causas dos riscos. Também, é realizada a definição da probabilidade dos controles atuais em detectar as causas antes do risco ocorrer. Propõe-se a utilização dos critérios de avaliação para os mecanismos de detecção conforme mostrado no Quadro 4. 
Quadro 4 - Escala de detecção (D).

\begin{tabular}{|c|l|c|}
\hline \multicolumn{2}{|c|}{ Probabilidade de deteç̃ão (D) } & Escala \\
\hline Remota & O controle atual não ou provavelmente não irá detectar o risco & 5 \\
\hline Baixa & Baixa probabilidade de o controle atual detectar o risco & 4 \\
\hline Moderada & O controle atual pode detectar o risco & 3 \\
\hline Alta & Alta probabilidade de o controle atual detectar o risco & 2 \\
\hline Muito alta & Quase certo que o controle atual irá detectar o risco & 1 \\
\hline
\end{tabular}

Fonte: Adaptado (FOGLIATTO, et al., 2009).

Em seguida, tem-se a atividade 3.2 que consiste na priorização dos riscos, cujo objetivo é selecionar os riscos que sejam mais críticos para o laboratório na fase de operação.

São propostas a realização das seguintes tarefas:

- Tarefa 3.2.1 - Calcular o NPR dos riscos levantados: após classificar cada risco de acordo com a severidade (S) dos efeitos, probabilidade de ocorrência (O) das causas e a probabilidade de detecção (D) das causas, deve-se multiplicar o valor de cada um desses elementos para calcular o NPR dos riscos levantados (S x O x D).

- Tarefa 3.2.2 - Determinar o peso relativo dos riscos: nesta sistemática propõe a utilização da divisão do NPR do risco prioritário considerado pela soma dos NPRs de todos os riscos calculados, como mostrado no Quadro 5.

Quadro 5 - Peso relativo dos riscos.

\begin{tabular}{|c|c|}
\hline Riscos & Peso relativo dos riscos \\
\hline$R_{1}$ & NPR $\left(\mathrm{R}_{1}\right) / \Sigma(\mathrm{NPR})$ \\
\hline $\mathrm{R}_{2}$ & $\mathrm{NPR}\left(\mathrm{R}_{2}\right) / \Sigma(\mathrm{NPR})$ \\
\hline $\mathrm{R}_{3}$ & $\mathrm{NPR}\left(\mathrm{R}_{3}\right) / \Sigma(\mathrm{NPR})$ \\
\hline $\mathrm{R}_{4}$ & $\mathrm{NPR}\left(\mathrm{R}_{4}\right) / \Sigma(\mathrm{NPR})$ \\
\hline $\mathrm{Rn}$ & $\mathrm{NPR}(\mathrm{Rn}) / \Sigma(\mathrm{NPR})$ \\
\hline
\end{tabular}

Fonte: adaptado Grubisic (2009). 
- Tarefa 3.2.3 - Determinar a estratégia de priorização (ponto de corte do NPR): o objetivo desta tarefa é definir quais riscos serão considerados prioritários por meio da definição de um ponto de corte. Considerando as escalas adotadas nos Quadro 14, Quadro 15 e Quadro 16,o valor do NPR pode variar de 1 a 125.

Segundo Fogliatto (2009), a equipe deve concentrar seus esforços nos riscos que possuírem os maiores NPR, nesse caso, nos riscos acima do ponto de corte. Para a execução dessa tarefa, propõe-se a utilização de um dos três métodos abaixo: o Valor médio: a estratégia de estabelecer um valor médio de NPR para definir o ponto de corte se dá pelo fato desse valor corresponder a uma situação intermediária de severidade, ocorrência e detecção (FOGLIATTO, et al., 2009). O valor médio desta sistemática corresponde a: 3 × 3 × $3=27$.

O Princípio de Pareto: segundo Fogliatto (2009), as equipes de GR podem ser mais ou menos rigorosas na avaliação da severidade, ocorrência e detecção. Sendo assim, atrelar o princípio de pareto faz mais sentido nesta situação, pois sua atuação acontecerá de fato sobre os riscos com maior NPR, independentemente do valor absoluto obtido.

- Tarefa 3.2.4 -Listar os riscos prioritários: após o cálculo do NPR, do ponto de corte adotado e da visualização dos riscos graficamente, deve-se verificar quais riscos serão priorizados e listá-los.

Por fim, tem-se a última atividade da Fase 3, Atividade 3.3 "Identificar as causas raízes", que consiste na investigação das causas dos riscos priorizados, ou seja, acima do ponto de corte. Para tal, propõe-se a utilização da técnica FTA.

A FTA tem o objetivo de identificar e analisar as causas que contribuem para o acontecimento de um evento indesejável. Esses fatores são identificados por dedução e organizados de uma maneira lógica e representados em um diagrama de árvore descrevendo seus fatores causais e sua relação lógica com o evento de topo (ABNT, 2012; IEC, 2006).

Neste trabalho, o evento topo (indesejável) equivale ao risco priorizado na tarefa anterior.

Após essa consideração, propõe-se a aplicação da técnica FTA ao longo da próxima tarefa. 
- Tarefa 3.3.1 - Aplicar a FTA para os riscos prioritários: a construção da árvore se iniciará partindo do risco indesejável (evento topo) e realizando um levantamento de suas causas. Após esta etapa, os eventos da árvore devem ser tipificados (intermediários, raiz, não explorados) e alocados segundo a relação de causa e efeito entre os eles, através dos conectores (Porta E e OU). Esta prática deverá ser replicada para cada risco prioritário, para que suas causas raízes sejam encontradas.

- Tarefa 3.3.2 - Listar as causas raízes levantadas: todas as causas raízes levantadas na tarefa anterior serão atualizadas no FMEA.

\subsection{Fase 4 - Tratamento de riscos}

O tratamento de riscos é a fase que tem como objetivo (i) definir ações para eliminar os riscos ou diminuir a probabilidade de ocorrência dos riscos e/ou a gravidade dos seus efeitos, (ii) definir os responsáveis por tratar os riscos e (iii) prazos para a implementação das ações para o tratamento dos riscos. Para isso, são propostas três atividades:

4.1 Planejar ações de tratamento de riscos,

\subsection{Priorizar ações e}

4.3 Implementar ações com suas respectivas entradas e saídas, conforme apresentado na Figura 9. 


\section{TRATAMENTO DE RISCOS}

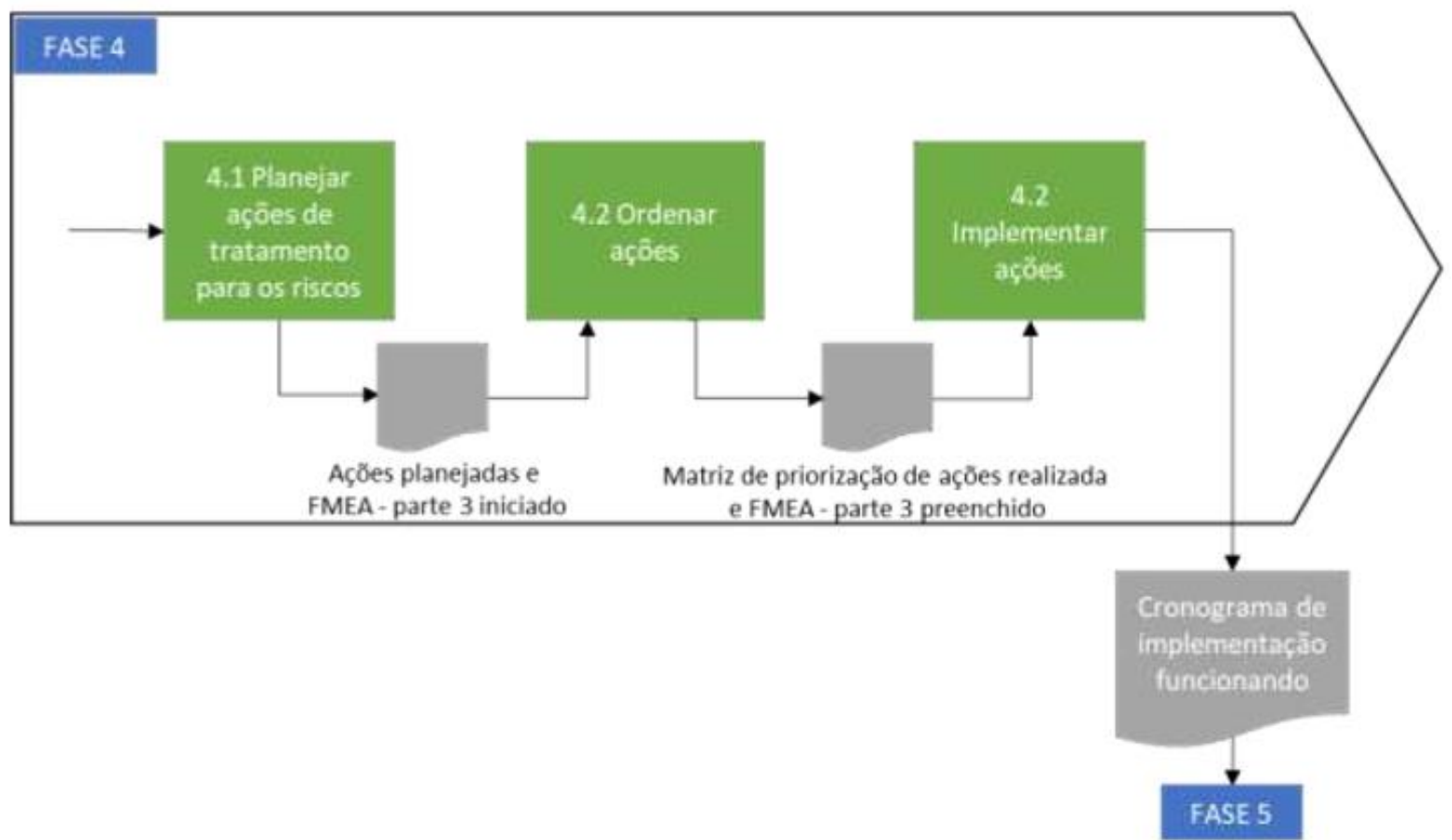

Figura 9 - Fase 4: Tratamento de riscos. Fonte: SILVA, 2019.

Para a atividade 4.1 - Planejar ações de tratamento para os riscos, foram definidas duas tarefas:

- Tarefa 4.1.1 - Propor ações: nesta tarefa são propostas orientações com o objetivo de auxiliar a identificação de ações para o tratamento dos riscos prioritários,

- Tarefa 4.1.2 - Definir responsáveis: esta tarefa consiste em definir quem será o responsável por cada ação, ou seja, quem cuidará da implementação e consequentemente do seu monitoramento e controle. Os nomes dos responsáveis serão colocados na coluna correspondente do FMEA modelo, localizado no Apêndice E.

A atividade 4.2 - Priorizar ações: baseada na Matriz de priorização de ações proposta por Grubisic (2009). Essa matriz tem como objetivo priorizar as ações que apresentam maior efeito no tratamento dos riscos prioritários de forma que essas ações sejam destacadas e 
implementadas primeiro. Para a realização desta etapa, é necessário puxar o peso relativo calculado na Tarefa 3.2.2.

- Tarefa 4.2.2 - Ordenar ações de tratamento: esta tarefa tem a finalidade de definir a ordem em que as ações serão implementadas.

- Tarefa 4.2.3 - Listar ações priorizadas: após o cálculo do NPA, ordenar as ações propostas do maior valor para o menor e organizá-las na FMEA.

Por fim, tem-se a atividade 4.3 - Implementar ações que consiste na implementação das ações por ordem de prioridade, cujo objetivo é garantir que as ações planejadas sejam executadas. Sendo assim, propõe-se a realização da seguinte tarefa:

- Tarefa 4.3.1 - Montar cronograma de implementação: de acordo com a ordem de execução das ações, deve-se elaborar um cronograma de implementação. Para realização desta tarefa, sugere-se a utilização de planilhas eletrônicas ou softwares de gerenciamento de projetos ou preencher os seguintes campos do FMEA: Data de início e Conclusão .

\section{CONCLUSÕES}

O presente artigo propôs uma sistemática de pesquisa com abordagem qualitativa e pronta para ser aplicada. A sistemática sugerida é capaz de, através da realização dos passos indicados, proporcionar um gerenciamento de riscos eficiente para a operação de um laboratório aberto. Tal metodologia foi aplicada no Laboratório Aberto da UnB e os resultados foram positivos.

A sistemática proposta busca contribuir com disseminação da cultura de riscos, característica que impacta de forma positiva na sua aplicação.

\section{REFERÊNCIAS}

ASSOCIAÇÃO BRASILEIRA DE NORMAS TÉCNICAS. NBR 31000: Gestão de Riscos Princípios e Diretrizes. RJ, 2009.

ASSOCIAÇÃO BRASILEIRA DE NORMAS TÉCNICAS. NBR 31010: Gestão de Riscos Técnicas para o processo de avaliação de riscos. RJ, 2012.

BARALDI, Paulo. Gerenciamento de Riscos Empresariais. Timburi/sp: Cia do Ebook, 2018. 
COMMITTEE OF SPONSORING ORGANIZATIONS OF THE TREADWAY COMMISSION. COSO: Enterprise Risk Management - Integrating with Strategy and Performance. 2017. Disponível em: 〈http://www.erm.coso.org>. Acesso em: 18 dez. 2018. FOGLIATTO, Flávio Sanson; RIBEIRO, José Luis Duarte. Confiabilidade e Manutenção Industrial. RJ: Elsevier, 2009.

GRUBISIC, V. V. F. Metodologia de Gerenciamento Integrado de Riscos Técnicos e Gerenciais para o Projeto de Produtos. 2009. 210 f. Tese (Doutorado) - Engenharia Mecânica, UFSC, Florianópolis, 2009.

MIKHAK, B., LYON, C., GORTON, T., GERSHENFELD, N., MCENNIS, C., and TAYLOR, J. Fab Lab: An alternate model of ICT for development, 2002.

PRINCE 2 (Ed.). Managing Successful Projects with PRINCE 2. London: The Stationary Office, 2002.

PROJECT MANAGEMENT INSTITUTE (Ed.). Um Guia do Conhecimento em Gerenciamento de Projetos (Guia PMBOK). 6. ed. Newtown Square: Project Management Institute, 2017.

SILVA, R. B. Sistemática para o Gerenciamento de Riscos em Laboratórios Avertos na Fase de Operação. 2019. 103f. TCC (Graduação) - Engenharia de Produção, FT, UnB, Brasília, 2019. SANTOS, A. C.; PIRES, F.; VIANA, D.M. Educação Empreendedora em Engenharia: Situação Atual e Perspectivas no Contexto Brasileiro/Implantação do Laboratório Aberto de Brasília. In: Adriana Maria Tonini. (Org.). DESAFIOS DA EDUCAÇÃO EM ENGENHARIA: Formação acadêmica e atuação profissional, Práticas Pedagógicas e Laboratórios Remotos. 1ed. Joinville: ABENGE, 2017. p. 98-103.

WILCZYNSKI, Vincent; ADREZIN, Ronald. Higher education makerspaces and engineering education. In: ASME 2016 International Mechanical Engineering Congress and Exposition. American Society of Mechanical Engineers, 2016.

ZIMMERMANN, Ana Carolina. Proposição de ambiente de aprendizagem ativa: Laboratório Aberto de Brasília. 2018. 155 f. TCC (Graduação) - Engenharia de Produção, FT, UnB, Brasília, 2018. 